\title{
Advances in nanopore sensing promises to transform healthcare
}

\author{
International Conference on Nanopore Technology (Shenzhen) \\ 30 March-1 April 2017, Shenzhen, China
}

The International Conference on Nanopore Technology (Shenzhen) was held from 30 March to 1 April 2017 in Shenzhen, China. The goal of the meeting was threefold: leverage the unique properties of nanopore technology to promote transformative advances in medicine, encourage cross-disciplinary collaborations in the research community within China and abroad; and discuss critical challenges that need to be addressed to rapidly advance the field. The meeting was chaired by Peixuan Guo, Endowed chair professor and Director of The Center for RNA Nanobiotechnology \& Nanomedicine at The Ohio State University, USA and co-chaired by Xian-En Zhang, distinguished professor of the Institute of Biophysics, Chinese Academy of Sciences, China. The conference was attended by more than 300 academic researchers, hospital administrators, government leaders and scientists from many disciplines across the country from both academic institutions and industry.

First draft submitted: 12 June 2017; Accepted for publication: 28 June 2017;

Published online: 27 July 2017

Keywords: nanotechnology • nanopore $\bullet$ Phi29 $\bullet$ MspA

Nanopores are nanometer-sized holes in a substrate. There are two types of nanopores: biological pores composed of proteins or nucleic acids embedded in lipid or polymer membranes, and synthetic pores fabricated in solid substrates. The detection method is electrical monitoring of ion-flow through the pore, as single molecules pass or bind/ interact with the pore. Both nanopore platforms have progressed rapidly over the last decade and significant advances have been made toward DNA and RNA sequencing, early disease diagnosis and a variety of other applications, as reported in a number of talks by leading researchers in the field. Oral presentations were organized into several themes: China government initiative, nanopore sequencing, technological advances, biophysical studies of macromolecule trans- port across nanopores and early disease diagnosis.

\section{China government initiative}

China's 13th Five-Year Plan has proposed to vigorously develop genome sequencing technologies for various diseases, highly-sensitive detection technologies, tumor targeted diagnosis and treatment methods. In response to this federal government initiative, local Shenzhen government sent several highprofile government leaders from Shenzhen municipality and Longhua District Government including Shenzhen Science and Technology Innovation Committee, Health and Family Planning Commission, and Development and Reform Commission. The leaders emphasized the need to continuously innovate to stay at the forefront of technol-
Farzin Haque ${ }^{*, 1,2}$, Shaoying Wang $* *, 1,2$, Taoxiang $\mathrm{Wu}^{1,2}$ \& Peixuan $\mathrm{Guo}^{3}$

'P\&Z Biological Technology, Newark, NJ, USA

2Shenzhen P\&Z Biomedical Company Limited, Shenzhen, China

${ }^{3}$ College of Pharmacy, Division of Pharmaceutics \& Pharmaceutical Chemistry, College of Medicine, Dorothy M. Davis Heart \& Lung Research Institute, $\mathrm{NCl}$ Comprehensive Cancer Center, Center for RNA Nanobiotechnology \& Nanomedicine, The Ohio State University, Columbus, $\mathrm{OH}$, USA

${ }^{*}$ Author for correspondence: farzin.haque@pzbiologyusa.com

**Author for correspondence:

shaoying.wang@pzbiologyusa.com

$\begin{array}{ll}\text { Future } & \text { fSg } \\ \text { Medicine } & \text { part of }\end{array}$ 
ogy development and reinforced government resource support of enterprises working with cutting-edge technologies.

\section{Nanopore sequencing}

One of the major advantages of nanopore sequencing is the feasibility of sequencing the genome in a short time frame and under US $\$ 1000$ per genome. The concept is rather simple: as a DNA strand traverses through the pore under an applied voltage, individual bases are identified based on modulations of ion current. However, there were several bottlenecks, such as speed of translocation, spatial and temporal resolution, biophysical properties of the pore and instrumentation. Jens Gundlach (University of Washington, USA) is leading the way of nanopore-based strand-sequencing using MspA nanopores. An enzyme (Phi29 DNA polymerase of helicase) is bound tightly to the singlestranded DNA and in the process, ratchets the DNA through the pore, one nucleobase at a time. However, base calling accuracy needs improvements as several bases in the narrow sensing region of the pore influence the electrical signal and also the enzyme turnover is stochastic. Gundlach's group is developing sophisticated bioinformatics approaches to address these issues toward more accurate nanopore sequencing. One prospective application is whole exosome sequencing as point of care technology. Cynthia Carnes (The Ohio State University, USA) highlighted the need for a rapid low-cost whole exosome sequencing technique that can be integrated with established clinical platforms to understand human arrhythmia pathophysiology and disease treatment.

\section{Technological advances}

Marija Drndic (University of Pennsylvania, USA) discussed how advances in ultra-thin solid substrates, device designs and new electronics have improved the temporal and spatial resolution of nanopores exemplified by fabrication of nanoribbons and field-effect transistors. Jingwei Bai (Tsinghua University, China) showed some recent developments in side-wall imagetransfer process for fabricating scalable solid state nanopore devices and tunneling gap devices. Yitao Long (East China University of Science and Technology, China) combined optical and electrical readout signal using novel plasmonic nanopores. Enhanced scattering of light can be monitored through a nanopore on gold film using dark-field microscopy to characterize single molecules at unprecedented details. Shuo Huang (Nanjing University) demonstrated optical sensing of nucleic acids in a high-throughput manner. An array of 2500 single droplet interfaced bilayer can be generated in a micropatterned hydrogel chip for characterizing nucleic acid sequence specific binding events in parallel. Deqiang Wang (Chinese Academy of Sciences) developed a scalable method of fabricating graphene nanopores with a helium ion microscope. Compared with conventional nanofabrication techniques, this method is faster and allows precise control of size and shape of the synthetic pores.

\section{Biophysical studies of macromolecule transport across nanopores}

Meni Wanunu (Northeastern University, USA) highlighted fundamental properties of nanopores, such as pore charge, dimension, pressure and electric fields, and how these factors influence transport of charged polymers in small confinements. Peixuan Guo presented highlights of his research using a robust nanochannel derived from bacteriophage phi29 DNA packaging motor. The channel represents one of the largest biological pores embedded in a lipid membrane. The channel can translocate single stranded and double stranded nucleic acids as well as peptides of various composition and length. Studying heterogenous and transient systems is difficult using conventional biochemical techniques. Shaoying Wang (P\&Z Biological Technology, NJ, USA) demonstrated how nanopores can detect peptide oxidation states in real-time at single molecule level. Similarly, Qing Zhao (Peking University, USA) used synthetic nanopores for determining the time-dependent kinetics of $\alpha$-synuclein oligomerization that has been implicated in the pathogenesis of Parkinson's disease. Zuhong Lu (Southeast University) presented advances in developing a hybrid device composed of a solid state pore with a fixed protein machine to study the dynamic properties of a single protein using horseradish peroxidase as an example. Yitao Long (East China University of Science and Technology, China) showed that aerolysin nanopore can resolve 2-nt oligonucleotides and can monitor the cleavage of oligonucleotides by exonuclease-I. His lab also developed a simultaneous optical and electrical detection method for studying different conformations of fluorescently labeled DNA through quartz nanopores. Haichen Wu (Chinese Academy of Sciences) used DNA probes, such as aptamers for sensing metal ions, small organic molecules and biopolymers using $\alpha$-hemolysin channels. Jia Geng (Sichuan University, China) developed synthetic analogs of biological membrane channels using carbon nanotubes and inserted them into a lipid bilayer as well as cell membrane to serve as a nanopore for transport of ions and biomolecules.

\section{Early disease diagnosis}

Early detection is critical for treating many types of diseases, including cancer, bacterial and viral infec- 
tions, and cardiovascular diseases among others. Liquid biopsy of patient body fluids is a rapidly emerging method for detecting biomarkers. While sequencing of DNA is one approach relying on the target molecule passing through the pore, another approach is to functionalize the terminal ends of the pores with programmable probes that can bind a target molecule with high specificity and sensitivity. Farzin Haque (P\&Z Biological Technology) presented the concept of capture and fingerprinting of single molecules using nanopores. Programmable receptors can be conjugated in the interior of phi29 nanopores for capturing single chemicals with high specificity and sensitivity. Peptide probes can also be engineered at the terminal ends of the phi29 channel to capture specific antibodies in the presence of many contaminants in the patient serum. Xinghua Lu (Chinese Academy of Sciences) demonstrated how synthetic nanopores can be used to detect DNA origami probes, bound to specific segments of DNA, for genomic diagnosis.

\section{Conclusion}

The conference highlighted how advances in nanopore are making new avenues for translating basic science into practical and clinical applications. A round table closed discussion session was held among the invited speakers to brainstorm fundamental challenges and discuss collaborative efforts to solve these challenges.

\section{Financial \& competing interests disclosure}

$F$ Haque and S Wang are employees of P\&Z Biological Technology. P Guo is a consultant for Oxford Nanopore, Nanobiodelivery Pharmaceutical Co. Ltd and P\&Z Biological Technology. The authors have no other relevant affiliations or financial involvement with any organization or entity with a financial interest in or financial conflict with the subject matter or materials discussed in the manuscript apart from those disclosed.

No writing assistance was utilized in the production of this manuscript. 